\title{
A dieta cetogênica pode auxiliar no tratamento do câncer? Uma análise crítica
}

\author{
Can the ketogenic diet help in cancer therapy? A critical \\ analysis.
}

\author{
Fabiana Neves Figueiredo', Vitor De Salles Painelli² \\ 'Instituto de Ciências da Saúde, Universidade Paulista \\ ${ }^{2}$ Escola de Artes, Ciências e Humanidades, Universidade de São Paulo \\ Contato: Vitor De Salles Painelli - vitor.painelli@gmail.com
}

\begin{abstract}
Resumo
Existe um crescente interesse na eficácia terapêutica da dieta cetogênica, dada a hipótese de que a restrição de carboidratos ( $\mathrm{CHO}$ ) favoreceria uma menor atividade de células tumorais. Mesmo assim, tais dietas continuam sendo um tema controverso na área de oncologia. Assim, este trabalho tem como objetivo sumarizar os estudos que já investigaram os potenciais efeitos terapêuticos da dieta cetogênica no tratamento do Câncer em pacientes humanos. Foi realizada uma revisão narrativa da literatura, nas bases de dados Literatura LatinoAmericana e do Caribe (LILACS), Scientific Electronic Library Online (SciELO), Sistema Online de Busca e Análise de Literatura Médica (MEDLINE) e base Centro Latino Americano e do Caribe de Informação em Ciências da Saúde (BIREME). As seguintes palavras-chaves foram utilizadas isoladamente ou em associação: câncer, nutrição, restrição de carboidrato, células cancerígenas, e dieta cetogênica. Artigos publicados entre 2010 e 2018 e nos idiomas Inglês e Português foram selecionados. Nenhum estudo clínico randomizado foi encontrado. Ao longo dos estudos revisados, os quais se incluíam em sua maioria desenhos quasiexperimentais e estudos de caso, 221 pacientes foram selecionados. Resultados mistos foram observados, tanto apoiando quanto refutando a eficácia terapêutica da dieta cetogênica no tratamento de pacientes com câncer. Importantemente, apenas 92 (42\%) desses pacientes foram submetidos à dieta cetogênica, dos quais somente $41 \%$ tiveram boa aderência à dieta. Estudos clínicos randomizados, investigando grandes segmentos de pacientes por longos períodos, com metodologias e protocolos dietéticos comparáveis, ainda são necessários antes que tal dieta possa ser recomendada como tratamento complementar do Câncer.
\end{abstract}

Palavras-chave: Carboidrato. Câncer. Restrição.

\begin{abstract}
There is a growing interest in the therapeutic efficacy of the ketogenic diet, given the hypothesis that carbohydrate restriction (CHO) would favor a decreased tumor cell activity. Nevertheless, such diets continue to be a controversial topic in the oncology field. Thus, this manuscript aimed to summarize the studies that have already investigated the potential therapeutic effects of the ketogenic diet in the treatment of cancer in human patients. A narrative review was carried, considering the databases Latin American and Caribbean Literature databases
\end{abstract}


(LILACS), the Scientific Electronic Library Online (SCiELO), the Online System of Search and Analysis of Medical Literature (MEDLINE) and the Latin American and Caribbean Health Sciences Information (BIREME). The following keywords were applied alone or in combination: cancer, nutrition, carbohydrate restriction, cancer cells and ketogenic diet. Articles published between 2010 and 2018, both in the English and Portuguese languages were selected. No randomized clinical trial was found. Throughout the reviewed studies, which included mostly quasiexperimental designs and case reports, 221 patients were selected. Mixed results were observed, both supporting and refuting the therapeutic efficacy of the ketogenic diet in the treatment of cancer patients. Importantly, only 92 (42\%) of these patients were submitted to ketogenic diets, of which only $41 \%$ had a good adherence to the diets. Randomized clinical trials investigating large patient segments over long periods with comparable dietary methodologies and protocols are still needed before such a diet can be recommended as a complementary treatment for cancer.

Keywords: Carbohydrate. Cancer. Restriction.

\section{INTRODUÇÃO}

O Câncer é caracterizado por um conjunto de doenças as quais acarretam num crescimento acelerado e anormal das células de um tecido específico, o qual pode alastrar-se para um ou mais diferentes tecidos ${ }^{1}$. A incidência do câncer no Brasil avança num ritmo acelerado, assim como em todo o mundo. Segundo o Instituto Nacional de Câncer José Alencar Gomes da Silva², até 2030, são esperados aproximadamente 21,4 milhões de novos casos de câncer, com 13,2 milhões de mortes em todo o mundo.

Mediante este quadro, a literatura tem se dedicado a examinar com maior profundidade de que maneira os fatores ambientais em específico, os hábitos alimentares, poderiam explicar esta crescente incidência do Câncer. Nesse sentido, o Câncer tem sido consistentemente relatado como uma condição rara entre sociedades não civilizadas com características de caçadores/coletores ${ }^{3,4}$. Estudos utilizando isótopos estáveis estimam que cerca de 22 a 40\% do consumo calórico de tais populações deriva de carboidratos (CHO), enquanto 19 a 30\% deriva de proteínas ${ }^{5}$. Ressalta-se que estes consumos estão abaixo e acima, respectivamente, daquele atualmente preconizado por recomendações internacionais ${ }^{6}$. De fato, considerando o tempo desde a revolução neolítica, o qual significou a transição do forrageamento e do nomadismo para a agricultura e colonização, estamos falando de uma fração inferior a 1\% da história humana. Nesse 'curto período' de tempo, alterações drásticas nos hábitos alimentares humanos ocorreram, tais como uma 
mudança da "dieta do homem das cavernas" (consistindo principalmente de gordura, carne e apenas ocasionalmente raízes, bagas e outras fontes de $\mathrm{CHO}$ ) para uma dieta 'dominada' por CHO's facilmente digeríveis, derivados principalmente de grãos, como alimento básico ${ }^{7}$. Especula-se que esta mudança dietética possa ter ocorrido muito rapidamente para induzir adaptações adequadas dos genes que codificam as vias metabólicas humanas, abrindo margem para condições como o Câncer. Tal quadro se torna ainda mais perturbador se levarmos em consideração a drástica alteração de outros hábitos individuais igualmente importantes, como é o caso da atividade física, cujos níveis vem diminuindo gradualmente na população mundial ao longo dos últimos 100 anos $^{8}$. Nesse âmbito, a combinação da má alimentação (e.g., excessiva ingestão de $\mathrm{CHO}^{\prime}$ 's de alto índice glicêmico) com o sedentarismo vem sendo apontados como fatores ambientais importantes na incidência do Câncer na sociedade moderna.

Em adição às profundas mudanças dos hábitos alimentares e de vida ao longo dos últimos anos, sabe-se que o metabolismo de células normais se diferencia do metabolismo de células cancerígenas e outras células em proliferação ou em desenvolvimento. Em 1924, o vencedor do Prêmio Nobel Otto Warburg9 descobriu que a taxa de captação de glicose aumenta dramaticamente nas células cancerígenas, onde as mesmas são capazes de produzir adenosina trifosfato (ATP) através de uma alta taxa de glicólise, mesmo na presença de oxigênio e mitocôndrias, caracterizando o conhecido Efeito Warburg. Inclusive, dados pré-clínicos sugerem que a via da insulina, incluindo a insulina, o fator de crescimento semelhante à insulina (do inglês Insulin-like Growth Factor, IGF) e o receptor de IGF, podem estar associados à iniciação e à progressão ao câncer ${ }^{10}$. Por conta disso, dentre as adaptações que ocorrem, as células cancerígenas apresentam uma notável regulação positiva dos transportadores de glicose em sua superfície ${ }^{11,12} \mathrm{e}$, frequentemente, uma superexpressão de várias enzimas-chave da via glicolítica ${ }^{13,14}$. Levando-se em consideração o metabolismo glicolítico predominante das células cancerígenas, bem como o potencial papel dos CHO's na incidência e prevalência do Câncer, uma série de autores vêm sugerindo que a redução da quantidade de $\mathrm{CHO}^{\prime}$ s na dieta poderia suprimir, ou pelo menos retardar, a atividade do Câncer ${ }^{15}$.

Uma das dietas restritivas de CHO's que mais ganhou ênfase nesse cenário é a dieta cetogênica, a qual consiste na implementação de uma dieta de alto e baixo conteúdo de gorduras e carboidratos, respectivamente, se comparadas à recomendação internacional ${ }^{6}$, enquanto o teor de proteínas é mantido dentro do adequado. A 
proporção convencional entre gordura vs. CHO e proteína dessa dieta é de 4:1 e 3:1, respectivamente, o que possibilitaria uma distribuição de macronutrientes da seguinte maneira, aproximadamente: $90 \%$ de gordura, $2 \%$ de $\mathrm{CHO}$ e $8 \%$ de proteína $^{16}$. Tal dieta vêm recebendo especial atenção pois em alguns tipos de Câncer, as células tumorais não têm a capacidade de metabolizar corpos cetônicos, devido à disfunção mitocondrial e diminuição da expressão das enzimas necessárias para a utilização de cetonas ${ }^{17}$. Logo, além de reduzir os níveis circulantes de glicose, a justificativa para fornecer uma dieta rica em gorduras e pobre em CHO's no tratamento do Câncer inclui a indução de cetose, de modo que as células cancerígenas fiquem sem energia, enquanto as células normais adaptariam o seu metabolismo para usar os corpos cetônicos e sobreviver. Além disso, ao reduzir os níveis circulantes de glicose, os níveis de insulina e de IGF, os quais se tratam de importantes impulsionadores da proliferação de células cancerígenas, também reduziriam. Apesar da aplicação terapêutica da dieta cetogênica já ter sido iniciada e demonstrada em certas doenças ${ }^{18,19}$, o número de evidências investigando o potencial terapêutico das dietas cetogênicas no tratamento do Câncer em humanos é reduzido, e os seus resultados, controversos. Em virtude do mencionado, o presente trabalho tem como objetivo sumarizar as evidências literárias que já se dedicaram a investigar a influência da dieta cetogênica no tratamento do Câncer, na tentativa de derivar recomendações baseadas em evidências para aconselhar pacientes com Câncer com relação a este regime. Para tanto, entre novembro de 2018 e janeiro de 2019 foi executada uma busca nas bases de dados Literatura LatinoAmericana e do Caribe (LILACS), na Scientific Electronic Library Online (SciELO), no Sistema Online de Busca e Análise de Literatura Médica (MEDLINE) e na Centro Latino Americano e do Caribe de Informação em Ciências da Saúde (BIREME), usando as seguintes palavras-chaves isoladamente e combinadamente: "cancer", "oncology", "tumor metabolism", "nutrition", "treatment", "carbohydrate restriction", "cancer cells", e "ketogenic diet". Desta busca inicial, foram encontrados 936 artigos. Para serem selecionados, os estudos deveriam ter o seu tema relacionado com a temática desta revisão narrativa. Não foram selecionados os artigos que fossem resumos, revisões ou opiniões, que estivessem em um idioma diferente do inglês, ou que empregassem a dieta por vias diferentes da gastrointestinal (i.e., nutrição parenteral). Além disso, de modo a aumentar a validade externa dos achados, somente artigos conduzidos em humanos e adultos 
foram selecionados. Com isso, 11 artigos foram selecionados como de suficiente qualidade para serem incluídos nesta revisão narrativa (Tabela 1).

Tabela 1 - Características dos estudos investigando os efeitos terapêuticos da dieta cetogênica em pacientes com câncer

\begin{tabular}{|c|c|c|c|c|}
\hline Autores & $\begin{array}{l}\text { Tamanho } \\
\text { amostral }\end{array}$ & $\begin{array}{c}\text { Amostra sob } \\
\text { restrição de } \mathrm{CHO}\end{array}$ & Duração & \% adesão dietética \\
\hline Zuccoli et al. ${ }^{20}$ & 1 & 1 & 10 sem & $100 \%$ \\
\hline Schmidt et al. ${ }^{21}$ & 16 & 16 & 12 sem & $31 \%$ \\
\hline Fine et al. ${ }^{22}$ & 10 & 10 & 4 sem & $50 \%$ \\
\hline Schoeder et al. ${ }^{23}$ & 11 & 11 & $5 d$ & Não reportado \\
\hline Rieger et al. ${ }^{25}$ & 20 & 20 & 6 sem & $85 \%$ \\
\hline Champ et al. ${ }^{24}$ & 53 & 6 & $3-12 \mathrm{~m}$ & Não reportado \\
\hline Schwartz et al. 26 & 2 & 2 & 12 sem & $100 \%$ \\
\hline Branca et al. 27 & 1 & 1 & 3 sem & $85 \%$ \\
\hline Klement \& Sweeny ${ }^{29}$ & 6 & 6 & $32-73 \mathrm{~d}$ & Não reportado \\
\hline Jansen \& Walach ${ }^{28}$ & 78 & 7 & 40 sem & Não reportado \\
\hline Tan-Shalaby et al. 30 & 17 & 17 & 16 sem & $64 \%$ \\
\hline
\end{tabular}

Legenda: sem = semanas; $m$ = meses; $d$ = dias

\section{DIETA CETOGÊNICA E CÂNCER: ESTAMOS MESMO DIANTE DE UM NOVO TRATAMENTO?}

Num dos estudos pioneiros a investigar o potencial terapêutico da dieta cetogênica

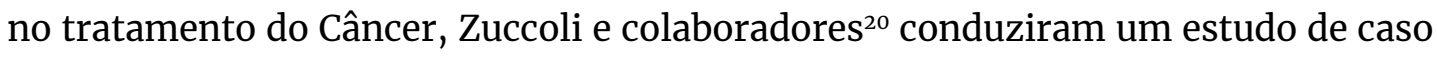
com uma paciente de 65 anos diagnosticada com glioblastoma multiforme, o tipo mais comum e agressivo de tumor maligno cerebral que acomete os seres humanos. Concomitantemente ao tratamento padrão (quimioterapia) da doença, durante dois meses, foi prescrita a esta paciente uma dieta cetogênica (4:1 de gordura:carboidrato + proteína) fornecendo $600 \mathrm{kcal} /$ dia. Após a intervenção, níveis reduzidos de glicose no sangue e níveis elevados de cetonas na urina foram verificados, atestando a eficácia da dieta em induzir cetose. No entanto, o resultado mais impressionante está relacionado ao fato de que, após a dieta, nenhum tecido tumoral cerebral discernível foi detectado usando imagens de ressonância magnética, e que imagens detectando a recorrência do tumor foram adquiridas 10 semanas após a suspensão da dieta. Dado que a rápida regressão desta doença é rara em pacientes idosos após o tratamento padrão isoladamente, a resposta observada nesse estudo de caso levou os autores a creem que a mesma provavelmente estava relacionada à ação da dieta cetogênica.

Estimulados por estes resultados encorajadores, num estudo piloto prospectivo e de desenho quase-experimental, Schmidt e colegas $^{21}$ recrutaram 16 pacientes (12 mulheres e 4 homens) em estágio tumoral avançado/metastático de tumores malignos sólidos de diferentes origens (e.g., ovário, pâncreas, tireoide), os quais não 
estavam passando por quimio- ou radioterapia, e os submeteram a 90 dias de dieta cetogênica (com menos de $90 \mathrm{~g}$ de carboidrato por dia). Um grande número de desistências ocorreu neste estudo, tornando inviável qualquer avaliação estatística do efeito da dieta sobre as características do tumor. Porém, verificou-se que o progresso da doença ocorreu em 5 pacientes que interromperam a dieta, enquanto 5 dos pacientes que aderiram à dieta durante todo o estudo mantiveram a doença estável. Ainda assim, fica difícil tirar qualquer conclusão com tal estudo considerando-se a baixa aderência dos pacientes à dieta $(31 \%)$, onde inclusive efeitos colaterais como constipação e fadiga foram relatados.

Com um desenho experimental e resultados bastante similares, Fine e colaboradores $^{22}$ empregaram uma dieta cetogênica de 26 a 28 dias, fornecendo carboidratos num máximo de $5 \%$ do consumo calórico diário, para 10 pacientes $(7$ mulheres e 3 homens) com diversos tipos de câncer em estado avançado e incurável. Mas mais uma vez, resultados mistos foram verificados, onde a doença permaneceu estável em 5 pacientes, progrediu em 4 enquanto 1 paciente apresentou uma remissão parcial da doença. E novamente, apenas 50\% dos participantes aderiu à dieta, onde efeitos colaterais relacionados à esta também incluíram constipação, fadiga e câimbras. Semelhante ao estudo acima citado, 11 pacientes com carcinoma de células escamosas de cabeça e de pescoço foram submetidos a 5 dias de uma dieta cetogênica (nenhuma especificação da mesma foi mencionada pelos autores), enquanto também eram submetidos à microdiálise para avaliação do metabolismo dos CHO's em tecidos tumorais e livre de tumor ${ }^{23}$. Como se tratou de um estudo intra-hospitalar, não ocorreram desistências do estudo. Também não foram detectados efeitos colaterais resultantes da dieta, apesar do curtíssimo tempo de intervenção. De forma interessante, os autores verificaram uma significante queda na concentração de lactato nas células tumorais após a adoção da dieta cetogênica, fato que ajudaria a sustentar a hipótese do efeito Warburg, onde os carboidratos representariam 'combustíveis' para o metabolismo glicolítico das células tumorais do carcinoma de cabeça e de pescoço. No entanto, nenhuma avaliação do tamanho dos tumores ou da atividade da doença foi realizada neste estudo.

Na sequência, três estudos prospectivos ${ }^{24}$, retrospectivos ${ }^{25}$ e estudo de caso $^{26}$, examinando o potencial terapêutico da dieta cetogênica em pacientes com glioblastoma multiforme foram conduzidos. Entre agosto de 2010 até abril de 2013, Champ e colegas ${ }^{24}$ analisaram o prontuário de pacientes diagnosticados com esta doença em estágio avançado e que realizavam regularmente a quimioterapia. Dos 53 
pacientes analisados, somente 6 de fato realizaram uma dieta cetogênica concomitante ao seu tratamento convencional, a qual visava fornecer um máximo de 30 a 50 g diárias de $\mathrm{CHO}^{\prime}$ s para os pacientes e $77 \%$ do consumo calórico diário na forma de gorduras. Apesar do heterogêneo tempo de duração ao qual cada um dos pacientes foi submetido à dieta (3 a 12 meses), a mesma se mostrou tolerável em todos eles. Num seguimento de 14 meses após o término das intervenções, os autores verificaram que apenas 2 dos 6 pacientes chegaram ao óbito. Contudo, análises referentes às características do tumor não foram executadas. Já Rieger e colaboradores $^{25}$ recrutaram 20 pacientes (13 mulheres e 7 homens) com glioblastoma multiforme recorrente, e concomitante à quimioterapia, os submeteram a 6 semanas de dieta cetogênica destinada a oferecer $60 \mathrm{~g}$ de CHO por dia. Em oposição a estudos anteriores, somente 3 pacientes não toleraram a dieta e desistiram do estudo (15\%), enquanto os outros não relataram quaisquer efeitos adversos relacionados à dieta. Mesmo assim, a doença se mostrou estável em apenas 3 pacientes e progressiva nos demais. Por fim, num estudo de caso, Schwartz e colegas $^{26}$ submeteram 2 homens com glioblastoma multiforme a 12 semanas de restrição calórica através da dieta cetogênica (na proporção de 3:1 de gordura:carboidrato + proteína) como monoterapia. De forma esperada, uma redução de peso corporal foi observada em ambos os pacientes. No entanto, também verificou-se uma progressão do tumor em ambos os pacientes, além do relato de dores de cabeça entre as semanas 6 e 8 de intervenção, as quais foram atribuídas à dieta.

Pouquíssimos estudos avaliaram o potencial terapêutico da dieta cetogênica isoladamente sobre um dos cânceres que mais atinge a população feminina, o câncer de mama. Num deles, Branca e colaboradores ${ }^{27}$ acompanharam uma mulher que teve um adenocarcinoma identificado no seio direito com a idade de 37 anos, e mesmo após a terapia convencional, 13 anos depois foi diagnosticada com o mesmo problema no seio direito. Apesar do tratamento convencional também ter sido aplicado a este segundo episódio, 15 anos após o mesmo, uma infiltração de adenocarcinoma foi identificada no seio direito novamente na porção que havia sido operada e submetida à radioterapia. Durante 3 semanas até a operação do mais recente episódio do adenocarcinoma, a paciente decidiu seguir por conta própria uma dieta cetogênica e a suplementação de vitamina D (10.000 IU/dia). Apesar de marcadores indiretos da doença terem sido reduzidos com a adoção da intervenção, não avaliou-se as características do tumor após a adoção desta. Tampouco é possível 
confirmar se os efeitos observados podem ser atribuídos apenas à dieta cetogênica ou à suplementação de vitamina $\mathrm{D}$, ou à combinação de ambos.

Os estudos prospectivos mais recentes sobre esta temática foram conduzidos por Jansen \& Walachv ${ }^{28}$, Klement \& Sweeney ${ }^{29}$ e Tan-Shalaby e colaboradores ${ }^{30}$. Jansen $\&$ Walach $^{28}$ recrutaram 78 pacientes com variados tipos de tumor (próstata, pulmão, mama) ou recorrência de tumor nos últimos 10 anos anteriores ao estudo, e os submeteram a 10 meses de intervenção. Porém, deste total de pacientes, somente 13 deles $(17 \%)$ de fato realizaram dietas cetogênicas. Se comparado aos outros pacientes, os autores demonstraram que este pequeno grupo de participantes que aderiu à dieta cetogênica diminuiu a expressão sanguínea de TKTL1, um novo marcador supostamente associado à progressão tumoral e à predominância do metabolismo glicolítico nas células tumorais ${ }^{31}$. Ainda assim, medidas diretas relacionadas às características do tumor não foram realizadas. Ademais, ressalta-se a ausência de um melhor relato das características da dieta cetogênica empregada, bem como da forma de controle à aderência da mesma. Com uma população amostral semelhante à de Jansen \& Walach ${ }^{28}$, Tan-Shalaby e colegas ${ }^{30}$ mobilizaram 17 pacientes com variados tipos de tumor (cerebral, pele, pancreático) em estágio avançado, e que não estavam sob quimioterapia, a 16 semanas de uma dieta cetogênica provendo 20 a 40 gramas de CHO diariamente. No entanto, apenas 11 participantes ( $\sim 64 \%$ ) aderiram à dieta cetogênica, dentre os quais 6,5 e 4 pacientes exibiram uma melhora na característica dos tumores com 4, 8 e 16 semanas, respectivamente. Melhoras significantes foram identificadas ao longo do estudo em questionários avaliando a qualidade de vida. Por outro lado, fadiga, constipação e insônia foram relatados frequentemente durante as primeiras 4 semanas de intervenção, mas reduziram substancialmente até o fim do estudo. Após o período de 16 semanas, somente 3 pacientes deram continuidade à dieta, dos quais 2 desenvolveram metástases cerebrais. Por fim, numa perspectiva similar a de um estudo de caso, mas com 6 pacientes, todos sob tratamento quimioterápico, e de diversificada idade, peso corporal, tipo (reto, mama, próstata) e estágio do tumor, Klement \& Sweeney ${ }^{29}$ avaliaram uma série de parâmetros clínicos em resposta a uma dieta cetogênica (70-80\% do consumo calórico diário proveniente de gorduras) de tempo variado para cada um dos pacientes (32 a 73 dias). Diferente dos outros estudos, a aderência à dieta foi estimulada por meio do fornecimento de livros educacionais e aconselhamento nutricional 1 vez por semana. Como resultados principais, nenhum efeito colateral grave relacionado à dieta ocorreu, a sensação 
subjetiva dos pacientes em relação à dieta foi classificada como boa, e a qualidade de vida avaliada através de questionários se manteve estável. Contudo, observou-se uma regressão do tumor em 5 pacientes em estágio inicial da doença, enquanto em 1 paciente com câncer de pulmão em estágio avançado foi verificada uma progressão lenta do tumor.

\section{LIMITAÇÕES DA LITERATURA}

Posicionamentos internacionais recomendam que a contribuição dos CHO's, das proteínas e das gorduras ao consumo calórico total seja de, respectivamente, $45 \%$ a $65 \%, 10 \%$ a $35 \%$, e $20 \%$ a $35 \%^{6}$, onde tais contribuições têm sido associadas a um risco reduzido de desenvolvimento de doenças crônicas e ao fornecimento adequado de nutrientes essenciais ${ }^{32}$. Distribuições de macronutrientes fora desta recomendação, no entanto, desafiam este conceito, como parece ser o caso da dieta cetogênica. A saber, a resposta fisiológica alcançada pela dieta cetogênica (caracterizada por um alto teor de gorduras, moderado a baixo conteúdo de proteínas, e muito baixo teor de $\mathrm{CHO}$ ) inclui a predominância do metabolismo de gorduras com uma limitada contribuição do metabolismo de CHO na produção de energia, consequentemente influenciando os níveis de $\mathrm{pH}$ sanguíneo, além de induzir elevados níveis de corpos cetônicos com uma concomitante redução dos níveis de glicose e insulina (i.e., indução de cetose). A adoção de dietas cetogênicas já demonstrou beneficiar a saúde em muitas condições patológicas, como a epilepsia $^{19}$, a obesidade ${ }^{33}$, o diabetes ${ }^{34}$ e doenças cardiovasculares ${ }^{35}$. Ainda assim, o potencial efeito terapêutico da dieta cetogênica em outras doenças de natureza metabólica, como o câncer, ainda requer maior exploração.

A presente revisão de literatura evidenciou a existência de poucos estudos empregando a dieta cetogênica como tratamento auxiliar para pacientes com câncer. A tabela 1 inclui uma visão ampla dos 11 estudos encontrados de acordo com a metodologia definida (publicações entre 2010-2018), onde estão incluídos 2 estudos de caso, 7 estudos prospectivos, e 2 estudos retrospectivos. Nenhum estudo com desenho metodológico rigoroso foi encontrado nos últimos 10 anos e resultados inconsistentes foram observados. Uma das razões que pode ajudar a explicar este quadro está relacionada ao baixo número de pacientes submetidos à intervenção. Apesar da presença de 215 pacientes somados em todos os estudos, apenas 97 (42\%) de fato foram submetidos a uma dieta cetogênica. Em outras palavras, os estudos são limitados por seus tamanhos amostrais. Além disso, a maioria dos estudos se 
preocupou apenas em testar a viabilidade, qualidade de vida e adesão do paciente à dieta cetogênica, e não avaliou ou não pôde avaliar o efeito antitumoral desta estratégia; enquanto aqueles que o avaliaram pecam pela falta de homogeneidade no tipo, localização e estágio do câncer, dificultando a comparação entre os resultados.

A baixa duração da intervenção em alguns dos estudos também é apresentada como uma potencial limitação da literatura, o que pode não ter sido o suficiente para permitir a cetoadaptação (i.e., depleção de estoques de glicogênio; aumento dos níveis de corpos cetônicos; aumento inicial seguido de eventual diminuição na gliconeogênese - paralela à redução na necessidade fisiológica de glicose; nível de glicose sanguínea um intervalo baixo-normal; e níveis diminuídos de triglicerídeos), bem como diferenças detectáveis no tamanho e metabolismo do tumor. Um outro ponto pouco explorado na atual literatura diz respeito à avaliação do estado de cetose, através da mensuração dos níveis sanguíneos de corpos cetônicos, os quais serviriam como um marcador importante de adesão à intervenção dietética. Inclusive, a baixa aderência à dieta cetogênica foi um importante problema detectado no presente manuscrito. Na verdade, a baixa taxa de aceitação de dietas restritivas já foi descrita na literatura ${ }^{36}$. Apoiando este ponto, uma meta-análise examinando a eficácia terapêutica de dietas cetogênicas no tratamento da epilepsia ${ }^{37}$, que incluiu 19 estudos observacionais com 1084 pacientes pediátricos, verificaram que $29,6 \%$ dos 552 desistentes interromperam a dieta devido à restritividade e/ou efeitos colaterais da dieta. Klein e colegas ${ }^{38}$ encontraram taxas ainda mais altas de descontinuidade da dieta entre adultos com epilepsia intratável: $51 \%$ dos pacientes sob dieta cetogênica tradicional e $42 \%$ dos pacientes sob a dieta de Atkins interromperam a dieta antes da conclusão do estudo. A maioria dos pacientes, mesmo aqueles com $75-100 \%$ de redução de frequência de convulsões, acabaram por interromper a dieta devido à restritividade e complexidade da dieta ${ }^{38}$. A partir do presente manuscrito, apenas 78 dos 97 pacientes $(80,41 \%)$ aderiram à prescrição dietética. Os pacientes restantes não cumpriram a restrição alimentar. Em adição à complexidade da dieta, efeitos colaterais foram relatados nos estudos revisados. Contudo, particularmente entre os pacientes com câncer, os efeitos colaterais podem não ser necessariamente atribuídos ao regime dietético, mas, erroneamente, podem ser considerados como efeitos colaterais da terapia medicamentosa ou da progressão da doença.

Por fim, os estudos nesta área não utilizaram protocolos dietéticos padronizados claramente comparáveis e consistentes. Em alguns estudos, a dieta do paciente 
sequer foi supervisionada por um nutricionista registrado. Em vez disso, os pacientes receberam instruções e um conjunto de folhetos com receitas de amostra e fatos sobre comida, enquanto outros forneceram protocolos detalhados, que poderiam ser replicados em pesquisas futuras.

\section{CONSIDERAÇÕES FINAIS}

Os estudos descritos neste manuscrito examinaram os efeitos de dietas cetogênicas em pacientes com Câncer. Em contraste à considerável atenção que pesquisadores, médicos e a mídia têm dado ao potencial terapêutico desta dieta sobre o Câncer, ainda faltam evidências científicas robustas apoiando os benefícios sobre o desenvolvimento e progressão do tumor, bem como sobre a redução dos efeitos colaterais resultantes das terapias convencionais do Câncer (i.e., quimioterapia; radioterapia). O escasso número de estudos e a grande heterogeneidade inerente aos desenhos experimentais e características de cada um deles (ex: diversificada duração da intervenção, bem como do tamanho e características da amostra; ausência de um grupo controle; informações precisas sobre a composição e acompanhamento da dieta; padronização do tipo estágio e tratamento do tumor sendo investigado; padronização das metodologias e técnicas usadas para avaliar os resultados) contribuem para uma baixa qualidade geral das evidências, limitando a capacidade de extrair conclusões assertivas sobre o assunto, e obviamente, de se estabelecer recomendações. Para tanto, estudos clínicos randomizados, com um grupo controle bem planejado, e metodologias consistentes e reprodutíveis, ainda são imprescindíveis para avaliar a real eficácia terapêutica da dieta cetogênica no tratamento de pacientes com Câncer, com especial atenção sendo dada aos possíveis efeitos colaterais resultantes da aplicação de tal dieta.

\section{REFERÊNCIAS}

1. Young VR. Energy metabolism and requirements in the cancer patient. Cancer Res. 1977; 37(7):2336-47.

2. ABC do câncer: abordagens básicas para o controle do câncer [internet]. Rio de Janeiro: INCA - Instituto Nacional de Câncer; [cited 2011]. Available from https://www.inca.gov.br/publicacoes/livros/abc-do-cancer-abordagensbasicas-para-o-controle-do-cancer.

3. Orenstein AJ. Freedom of Negro Races from Cancer. Br Med J. 1923; 2:342.

4. Brown GM, Cronk LB, Boag TJ. The occurrence of cancer in an Eskimo. Cancer. 1952; 5(1):142-3. 
5. Cordain L, Miller JB, Eaton SB, Mann N. Macronutrient estimations in huntergatherer diets. Am J Clin Nutr. 2000; 72(6):1589-92.

6. Thomas DT, Erdman KA, Burke LM. American College of Sports Medicine Joint Position Statement. Nutrition and Athletic Performance. Med Sci Sports Exerc. 2016; 48(3):543-68.

7. Carrera-Bastos P, Fontes-Villalba M, O'Keefe JH, Lindeberg S, Cordain L. The western diet and lifestyle and diseases of civilization. Res Rep Clin Card. 2011; 2:15-35.

8. Kohl HW 3rd, Craig CL, Lambert EV, Inoue S, Alkandari JR, Leetongin G, et al. The pandemic of physical inactivity: global action for public health. Lancet. 2012; 380(9839):294-305.

9. Warburg, O. Uber den Stoffwechsel der Tumoren. Berlin: Springer; 1926. Translated: The metabolism of tumors. London: Arnold Constable; 1930.

10. Vander Heiden MG, Cantley LC, Thompson CB. Understanding the Warburg effect: the metabolic requirements of cell proliferation. Science. 2009;324(5930):1029-33.

11. Lambert DW, Wood IS, Ellis A, Shirazi-Beechey SP. Molecular changes in the expression of human colonic nutrient transporters during the transition from normality to malignancy. Br J Cancer. 2002;86(8):1262-9.

12. Mathupala SP, Rempel A, Pedersen PL. Glucose catabolism in cancer cells: identification and characterization of a marked activation response of the type II hexokinase gene to hypoxic conditions. J Biol Chem. 2001;276(46):43407-12.

13. Moreno-Sanchez R, Rodríguez-Enríquez S, Marín-Hernandez A, Saavedra E. Energy metabolism in tumor cells. FEBS J. 2007;274(6):1393-418.

14. Cairns RA, Harris IS, Mak TW. Regulation of cancer cell metabolism. Nat Rev Cancer. 2011;11(2):85-95.

15. Brouns F. Overweight and diabetes prevention: is a low-carbohydrate-high-fat diet recommendable? Eur J Nutr. 2018;57(4):1301-12.

16. Allen BG, Bhatia SK, Anderson CM, Gilmore JM, Sibenaller ZA, Mapuskar KA, et al. Ketogenic diets as an adjuvant cancer therapy: History and potential mechanism. Redox Biol. 2014;2:963-70.

17. Morscher RJ, Aminzadeh-Gohari S, Feichtinger RG, Mayr JA, Lang R, Neureiter $\mathrm{D}$, et al. Inhibition of Neuroblastoma Tumor Growth by Ketogenic Diet and/or Calorie Restriction in a CD1-Nu Mouse Model. PLoS One. 2015;10(6):e0129802.

18. Stafstrom CE, Rho JM. The ketogenic diet as a treatment paradigm for diverse neurological disorders. Front Pharmacol. 2012;3:59.

19. Martin K, Jackson CF, Levy RG, Cooper PN. Ketogenic diet and other dietary treatments for epilepsy. Cochrane Database Syst Rev. 2016;2:CD001903.

20. Zuccoli G, Marcello N, Pisanello A, Servadei F, Vaccaro S, Mukherjee P, Seyfried TN. Metabolic management of glioblastoma multiforme using standard therapy together with a restricted ketogenic diet: case report. Nutr. Metab. (Lond.) 2010; 7:33.

21. Schmidt M, Pfetzer N, Schwab M, Strauss I, Kammerer U. Effects of a ketogenic diet on the quality of life in 16 patients with advanced cancer: a pilot trial. Nutr Metab (Lond). 2011; 8(1):54. 
22. Fine EJ, Segal-Isaacson CJ, Feinman RD, Herszkopf S, Romano MC, Tomuta N, et al. Targeting insulin inhibition as a metabolic therapy in advanced cancer: a pilot safety and feasibility dietary trial in 10 patients. Nutrition. 2012; 28(10):1028-35.

23. Schroeder U, Himpe B, Pries R, Vonthein R, Nitsch S, Wollenberg B. Decline of lactate in tumor tissue after ketogenic diet: In vivo microdialysis study in patients with head and neck cancer. Nutr Cancer. 2013; 65(6):843-9

24. Champ CE, Palmer JD, Volek JS, Werner-Wasik M, Andrews DW, Evans JJ, et al. Targeting metabolism with a ketogenic diet during the treatment of glioblastoma multiforme. J Neurooncol. 2014; 117(1):125-31.

25. Rieger J, Bahr O, Maurer GD, Hattingen E, Franz K, Brucker D, et al. ERGO: a pilot study of ketogenic diet in recurrent glioblastoma. Int J Oncol. 2014; 44(6):184352.

26. Schwartz K, Chang HT, Nikolai M, Pernicone J, Rhee S, Olson K, et al. Treatment of glioma patients with ketogenic diets: Report of two cases treated with an IRB approved energy-restricted ketogenic diet protocol and review of the literature. Cancer Metab. 2015;3:3.

27. Branca JJV, Pacini S, Ruggiero M. Effects of pre-surgical vitamin D supplementation and ketogenic diet in a patient with recurrent breast cancer. Anticancer Res. 2015; 35(10):5525-32.

28. Jansen $\mathrm{N}$, Walach $\mathrm{H}$. The development of tumours under a ketogenic diet in association with the novel tumour marker TKTL1: A case series in general practice. Oncol Lett. 2016; 11(1):584-92.

29. Klement RJ, Sweeney RA. Impact of a ketogenic diet intervention during radiotherapy on body composition: I. Initial clinical experience with six prospectively studied patients. BMC Res Notes. 2016; 9:143.

30. Tan-Shalaby JL, Carrick J, Edinger K, Genovese D, Liman AD, Passero VA, et al. Modified Atkins diet in advanced malignancies - final results of a safety and feasibility trial within the Veterans Affairs Pittsburgh Healthcare System. Nutr Metab (Lond). 2016; 13:52.

31. Wanka C, Steinbach JP, Rieger J. Tp53-induced glycolysis and apoptosis regulator (TIGAR) protects glioma cells from starvation-induced cell death by up-regulating respiration an dimproving cellular redox homeostasis. J Biol Chem. 2012; 287:33436-46.

32. de Souza RJ, Swain JF, Appel LJ, Sacks FM. Alternatives for macronutrient intake and chronic disease: A comparison of the OmniHeart diets with popular diets and with dietary recommendations. Am J Clin Nutr. 2008; 88(1):1-11.

33. Johnstone AM, Horgan GW, Murison SD, Bremner DM, Lobley GE. Effects of a high-protein ketogenic diet on hunger, appetite, and weight loss in obese men feeding ad libitum. Am J Clin Nutr. 2008; 87(1):44-55.

34. Boden G, Sargrad K, Homko C, Mozzoli M, Stein TP. Effect of a low carbohydrate diet on appetite, blood glucose levels, and insulin resistance in obese patients with type 2 diabetes. Ann Intern Med. 2005; 142(6):403-11.

35. Sharman MJ, Kraemer WJ, Love DM, Avery NG, Gómez AL, Scheett TP, et al. A ketogenic diet favorably affects serum biomarkers for cardiovascular disease in normal weight men. J Nutr. 2002; 132(7):1879-1885. 
36. Brouns F. Overweight and diabetes prevention: is a low-carbohydrate-high-fat diet recommendable? Eur J Nutr. 2018; 57(4):1301-12.

37. Henderson CB, Filloux FM, Alder SC, Lyon JL, Caplin DA. Efficacy of the ketogenic diet as a treatment option for epilepsy: meta-analysis. J Child Neurol. 2006; 21(3):193-8.

38. Klein P, Tyrlikova I, Mathews GC. Dietary treatment in adults with refractory epilepsy: a review. Neurology. 2014; 83(21):1978-85.

Submissão: 15/07/2019

Aprovação: 20/07/2020 